\title{
Clinical characteristics of Polish patients with ANCA-associated vasculitides-retrospective analysis of POLVAS registry
}

\author{
K. Wójcik ${ }^{1} \cdot$ K. Wawrzycka-Adamczyk ${ }^{1} \cdot$ A. Włudarczyk ${ }^{1} \cdot$ J. Sznajd $^{2,3} \cdot$ Z. Zdrojewski $^{4} \cdot$ A. Masiak $^{4} \cdot$ Z. Czuszyńska $^{4}$.

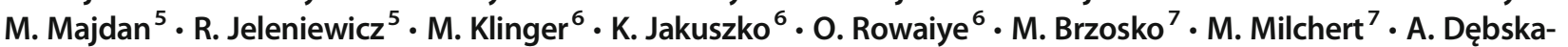

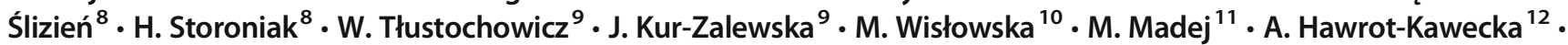 \\ P. Głuszko ${ }^{13}$. E. Kucharz ${ }^{14}$. Jacek Musiał ${ }^{1}$
}

Received: 20 December 2018 / Revised: 14 March 2019 / Accepted: 29 March 2019 / Published online: 23 April 2019

(C) The Author(s) 2019

\begin{abstract}
Objective Antineutrophil cytoplasmic antibody (ANCA)-associated vasculitides (AAV) are rare small to medium-size vessel systemic diseases. As their clinical picture, organ involvement, and factors influencing outcome may differ between countries and geographical areas, we decided to describe a large cohort of Polish AAV patients coming from several referral centers - members of the Scientific Consortium of the Polish Vasculitis Registry (POLVAS).

Methods We conducted a systematic multicenter retrospective study of adult patients diagnosed with AAV between Jan 1990 and Dec 2016 to analyze their clinical picture, organ involvement, and factors influencing outcome. Patients were enrolled to the study by nine centers (14 clinical wards) from seven Voivodeships populated by $22.3 \mathrm{mln}$ inhabitants $(58.2 \%$ of the Polish population).

Results Participating centers included 625 AAV patients into the registry. Their distribution was as follows: 417 patients (66.7\%) with GPA, $106(17.0 \%)$ with MPA, and $102(16.3 \%)$ with EGPA. Male-to-female ratios were almost 1:1 for GPA (210/207) and MPA (54/52), but EGPA was twice more frequent among women (34/68). Clinical manifestations and organ involvement were analyzed by clinical phenotype. Their clinical manifestations seem very similar to other European countries, but interestingly, men with GPA appeared to follow a more severe course than the women. Fifty five patients died. In GPA, two variables were significantly associated with death: permanent renal replacement therapy (PRRT) and respiratory involvement (univariate analysis). In multivariate analysis, PRRT ( $\mathrm{OR}=5.3 ; 95 \%$ confidence
\end{abstract}

Electronic supplementary material The online version of this article (https://doi.org/10.1007/s10067-019-04538-w) contains supplementary material, which is available to authorized users.

Jacek Musiał

jacek.musial@uj.edu.pl

1 2nd Department of Internal Medicine, Faculty of Medicine, Jagiellonian University Medical College, ul. Skawińska 8, 31-066 Kraków, Poland

2 Department of Rheumatology, Raigmore Hospital, Inverness, UK

3 Institute of Applied Health Sciences, University of Aberdeen, Aberdeen, UK

4 Department of Internal Medicine, Connective Tissue Diseases and Geriatrics, Medical University of Gdańsk, Gdańsk, Poland

5 Department of Rheumatology and Connective Tissue Diseases, Medical University of Lublin, Lublin, Poland

6 Department of Nephrology and Transplantation Medicine, Wroclaw Medical University, Wrocław, Poland

7 Department of Rheumatology and Internal Diseases, Pomeranian Medical University in Szczecin, Szczecin, Poland
8 Department of Nephrology, Transplantology and Internal Diseases, Medical University of Gdańsk, Gdańsk, Poland

9 Department of Internal Medicine and Rheumatology, Military Medicine Institute, Warszawa, Poland

10 Department of Internal Diseases and Rheumatology, Central Clinical Hospital of the Ministry of the Interior and Administration, Warszawa, Poland

11 Department of Rheumatology and Internal Medicine, Wroclaw Medical University, Wrocław, Poland

12 Department of Internal Medicine and Metabolic Diseases, Medical University of Silesia, Katowice, Poland

13 Department of Rheumatology, National Institute of Geriatrics, Rheumatology and Rehabilitation, Warszawa, Poland

14 Department of Internal Medicine and Rheumatology, Medical University of Silesia, Katowice, Poland 
interval $(\mathrm{CI})=2.3-12.2)$, respiratory involvement $(\mathrm{OR}=3.2 ; 95 \% \mathrm{CI}=1.06-9.7)$, and, in addition, age $>65(\mathrm{OR}=2.6$; 95\% CI $=1.05-6.6)$ were independently associated with death. In MPA, also three variables were observed to be independent predictors of death: PRRT $(\mathrm{OR}=5.7 ; 95 \% \mathrm{CI}=1.3-25.5)$, skin involvement $(\mathrm{OR}=4.4 ; 95 \% \mathrm{CI}=1.02-$ 19.6), and age $>65(\mathrm{OR}=6.3 ; 95 \% \mathrm{CI}=1.18-33.7)$.

Conclusions In this first multicenter retrospective study of the Polish AAV patients, we have shown that their demographic characteristics, disease manifestations, and predictors of fatal outcome follow the same pattern as those from other European countries, with men possibly suffering from more severe course of the disease.

Keywords Antineutrophil cytoplasmic antibodies $\cdot$ Churg-Strauss syndrome $\cdot$ Microscopic polyangiitis $\cdot$ Registries $\cdot$ Wegener's granulomatosis

\section{Introduction}

Vascultides are a heterogeneous group of rare diseases with unknown etiology and the clinical spectrum ranging from life-threatening systemic disease, through single organ involvement to minor isolated skin changes $[1,2]$. Present nomenclature and definitions of systemic vasculitides have been proposed in 2012 at the International Chapel Hill Consensus Conference (CHCC 2012) [3]. Among various forms of vasculitic syndromes (antineutrophil cytoplasmic antibody (ANCA)-associated vasculitides (AAV)), belong to the small to medium-size vessel systemic diseases comprising granulomatosis with polyangiitis (GPA, previously known as Wegener's granulomatosis (WG), microscopic polyangiitis (MPA), and eosinophilic granulomatosis with polyangiitis (EGPA, previously known as Churg-Strauss syndrome (CSS)). Despite progress made in the management of AAV, those patients still remain at an increased risk of death. Recent publication indicates that standardized mortality ratio in AAV patients is 2.7-fold higher compared with the general population [4].

Epidemiology and clinical characteristics of AAV have been described in several studies, most often, however, analyzing jointly GPA and MPA [5, 6] and considering both entities as a spectrum of the same pathological process. EGPA patients were usually analyzed separately [5, 7]. However, ANCA specificity, clinical phenotype, and outcome of AAV patients may differ depending on geographic area and ethnicity [8-10].

For this reason, we decided to study in a retrospective manner immunologic profile, clinical picture, and the disease outcome in ethically uniform Polish AAV patients depending on their phenotype. We also attempted to identify in these patients poor outcome predictors. As studies on Polish AAV patients were usually limited to one center, including single diagnosis and relatively small number of patients $[11,12]$, we established Scientific Consortium of the Polish Vasculitis Registry (POLVAS) [13-15] to increase substantially the number of analyzed subjects.

\section{Methods}

We conducted a multicenter retrospective study of all adult patients diagnosed with AAV by participating centers between 1990 and 2016. Their clinical and laboratory data were collected in POLVAS registry [13] by nine referral centers (14 clinical wards) from seven Voivodeships with $22.3 \mathrm{mln}$ inhabitants, encompassing $58.2 \%$ of the Polish population. Specialties of the centers included rheumatology (10), nephrology (2), metabolic diseases (1), and clinical immunology (1). The work described in this article has been carried out in accordance with The Code of Ethics of the World Medical Association (Declaration of Helsinki) for experiments involving humans; the locally appointed ethics committee of each partner has approved the research protocol, and informed consent has been obtained from the subjects (or their legally authorized representative). Study protocol was approved by Bioethical Commission of Jagiellonian University, decision No 122.6120.25.2016. Only patients who met the American College of Rheumatology (ACR) classification criteria for GPA(WG) or EGPA(CSS) and fulfilled requirements for the nomenclature of GPA, MPA, and EGPA according to CHCC 2012 [3] were included in the study. Specific organ involvement, disease relapse, and disease remission were defined retrospectively according to BVAS v3 [16]. Patients diagnosed with AAV according to medical documentation but lost to follow-up at the time of data collection were not included to the study.

Following data were systematically collected according to the common protocol: demographics, clinical, laboratory, radiographic, pathology, and treatment details. ANCA positivity in the earliest patients included into Registry (1990-2000) was detected by an indirect immunofluorescence (IIF) method only and identified as cANCA or pANCA positivity. Later, ANCA positivity was detected by both, ELISA (anti-PR3 or anti-MPO antibodies) and, if necessary, by IIF.

\section{Statistical analysis}

Categorical data were summarized as percentages; significant differences or associations were analyzed using the 
$\chi^{2}$ test or Fisher's exact tests. Continuous variables are presented as mean and/or median and interquartile range [IQR], depending on normality of data distribution demonstrated by Kolmogorov-Smirnov test. Associations of quantitative data were analyzed with Student's $t$ test and with the nonparametric Mann-Whitney $U$ test or the Kruskal-Wallis test. For analysis of risks related to mortality and the need for permanent renal replacement therapy (PRRT), variables shown to be statistically significant in the univariate analysis were further analyzed in a multivariate logistic regression model. Statistical analysis was performed using Statistica 13 software.

\section{Results}

\section{Demographic analysis of AAV patients}

Participating centers included a total of 625 adult AAV patients into our retrospective registry. All patients were uniformly Caucasian. Demographic data of our AAV patients and basic information about their ANCA status are given in Table 1. Among 625 patients, only 26 (4.2\%) were diagnosed between 1990 and 2000 with the remaining 599 subjects diagnosed after the year 2000 .

AAV patients from POLVAS registry included $66.7 \%$ diagnosed with GPA, $17.0 \%$ with MPA, and $16.3 \%$ with EGPA. As in other European countries, male-to-female ratio was almost 1:1 for GPA (210/207) and MPA (54/52). Unexpectedly, EGPA was diagnosed twice more often among women (Table 1).

\section{Demographic characteristics of AAV patients according to their ANCA specificity}

In our cohort, 127 subjects were identified with anti-MPO+ and 334 with anti-PR3+ (see Suppl. Tab.S1 and S2). Analysis by antibody specificity did not add important new information to the one obtained by phenotype analysis. Understandably, EGPA demographic characteristic significantly differed from that of MPA - main AAV group with anti-MPO+ antibodies. Surprisingly, the age at diagnosis in EGPA anti-MPO+ subgroup and male to female ratio (1:1) was similar to other antiMPO+ positive AAV patients (median age at diagnosis 56.9 years vs 63.1 years; $p=0.25$ ) (Suppl. Tab.S1 and S3).

\section{Clinical manifestations of AAV}

Organ involvement and laboratory parameters of different AAV phenotypes are summarized in Table 1. The main organs affected in GPA and EGPA were respiratory tract and ENT, whereas in MPA, kidneys followed by lower respiratory tract. EGPA was the entity characterized by the highest number of organs involved. Heart and neurological involvement was also highly represented in this group as compared to GPA and MPA. In terms of kidney function, the highest (ever documented) maximal serum creatinine concentration was observed in MPA patients, as was the need for temporary or permanent RRT.

Interestingly, $2.9 \%$ of patients with GPA (10 out of 342 in whom differential blood count was available) showed increased blood eosinophil count (defined as $>1500$ cells $/ \mathrm{mm}^{3}$ or $>10 \%$ of total leukocyte count).

Disease relapses were not only prevalent in GPA patients but also affected many EGPA subjects with the shortest time between diagnosis and the first relapse in the latter. Relapse rate (expressed as number of relapses per 100 patients/years) was higher among GPA (18.0) and EGPA (25.2) patients than in the MPA group (12.0) (Table 1). Of note, in our cohort, the average observation time in MPA group was significantly shorter (median 27.4 months) than in GPA (61.9 months) and EGPA (68 months).

Biopsy was performed in $381(61 \%)$ cases (renal-106, lung-65, muscle, nerve-1, ENT-115, skin-42, eye-5, muscle4 , intestine-9, other-31). The diagnosis of vasculitis was supported by histopathology in $310(81.4 \%)$ subjects.

\section{Factors related to poor outcome}

Among all AAV subjects who died during retrospective observation ( $n=55 ; 8.8 \%$ ), 42 suffered from GPA and 13 from MPA, which corresponds to $10.1 \%$ and $12.3 \%$ of patients, respectively (Table 2 ). AAV patients who died showed higher serum creatinine concentration ever observed, more often required permanent RRT, and suffered from multiorgan disease (Table 2).

The age of GPA patients who died did not differ from those who remained alive, but it appears that there were slightly more men (26 men vs 16 women), although this difference did not reach statistical significance. GPA patients who died showed more frequently respiratory and kidney involvement and were more prone to relapse, while MPA patients were older, and showed higher rate of skin and ENT involvement (Table 2).

Analysis of factors related to death in AAV identified various aspects of kidney involvement as a key health issue in both GPA and MPA. This prompted us to investigate which factors differentiated between patients requiring temporary or permanent renal replacement therapy from those who did not (Table 3).

In general, MPA patients significantly more often required RRT than the GPA ones ( $44 \%$ vs $21 \% ; p<0.0001)$. GPA patients who were never dialyzed $(330 ; 79 \%)$ were on average 7 years younger (47.6 years vs 54.3 years $p<0.001)$ and showed ENT involvement significantly more frequently than those dialyzed. Again, men prevailed among GPA patients 
Table 1 Demographic, clinical characteristics, and ANCA status of AAV patients in the POLVAS Registry

\begin{tabular}{|c|c|c|c|c|c|c|}
\hline & GPA & MPA & EGPA & $\begin{array}{l}\text { GPA vs } \\
\text { MPA }\end{array}$ & $\begin{array}{l}\text { GPA vs } \\
\text { EGPA }\end{array}$ & $\begin{array}{l}\text { MPA vs } \\
\text { EGPA }\end{array}$ \\
\hline No. of patients & 417 & 106 & 102 & $p$ & & \\
\hline Male/female & $210 / 207$ & $54 / 52$ & $34 / 68$ & NS & $<0.01$ & $<0.05$ \\
\hline Age (years); median, [range] & $51.4[13-80]$ & $63.7[19-85]$ & $44.5[9-85]$ & $<0.01$ & $<0.05$ & $<0.0001$ \\
\hline $\begin{array}{l}\text { ANCA positive }^{1} ; n(\%) \\
\text { ANCA negative; } n(\%)\end{array}$ & $\begin{array}{l}376(93.3) \\
26(6.7)\end{array}$ & $\begin{array}{l}104(98.1) \\
2(1.9)\end{array}$ & $\begin{array}{l}43(47.8) \\
47(52.2)\end{array}$ & NS & $<0.00001$ & $<0.00001$ \\
\hline ANCA-missing data/not performed & 15 & 0 & 12 & & & \\
\hline $\begin{array}{l}\text { cANCA/anti-PR3 }{ }^{2} \\
\text { pANCA/anti-MPO }\end{array}$ & $\begin{array}{l}344(85.6) \\
19(4.7)\end{array}$ & $\begin{array}{l}9(8.5) \\
87(82.1)\end{array}$ & $\begin{array}{l}10(11.1) \\
28(31.1)\end{array}$ & $<0.00001$ & $<0.00001$ & $<0.00001$ \\
\hline Double positive $^{2}$ & $13(3.2)$ & $6(5.7)$ & $2(2.2)$ & & & \\
\hline Time to diagnosis ${ }^{4}$ (month); median, [IQR] & $5.95[2.95-14.0]$ & $5.01[2-10.95]$ & $14.9[7.7-34.8]$ & NS & $<0.001$ & $<0.0001$ \\
\hline \multicolumn{7}{|l|}{ Organ involvement } \\
\hline General $n(\%)$ & $355(85.1)$ & $83(78.3)$ & $87(85.3)$ & NS & NS & NS \\
\hline Skin $n(\%)$ & $140(33.6)$ & $24(22.6)$ & $51(50)$ & $<0.05$ & $<0.01$ & $<0.001$ \\
\hline Eye $n(\%)$ & $114(27.3)$ & $4(3.8)$ & $6(5.9)$ & $<0.0001$ & $<0.0001$ & NS \\
\hline ENT $n(\%)$ & $315(75.5)$ & $18(17.0)$ & $82(80.4)$ & $<0.0001$ & NS & $<0.0001$ \\
\hline Respiratory $n(\%)$ & $315(75.5)$ & $60(56.6)$ & $100(98.0)$ & $<0.0001$ & $<0.0001$ & $<0.0001$ \\
\hline Heart $n(\%)$ & $55(13.2)$ & $11(10.4)$ & $43(42.2)$ & NS & $<0.0001$ & $<0.0001$ \\
\hline GI $n(\%)$ & $46(11.0)$ & $12(11.3)$ & $25(24.5)$ & NS & $<0.001$ & $<0.05$ \\
\hline Renal $n(\%)$ & $252(60.4)$ & $101(95.3)$ & $25(24.5)$ & $<0.0001$ & $<0.0001$ & $<0.0001$ \\
\hline Urinary $n(\%)$ & $8(1.9)$ & $3(2.8)$ & 0 & NS & NS & NS \\
\hline CNS $n(\%)$ & $45(10.8)$ & $2(1.9)$ & $8(7.8)$ & $<0.01$ & NS & $<0.05$ \\
\hline Neurological $n(\%)$ & $75(18.0)$ & $20(18.9)$ & $50(49.0)$ & NS & $<0.0001$ & $<0.0001$ \\
\hline Creatinine $\max ^{5}(\mathrm{mg} / \mathrm{dl})$; median, [IQR] & $1.04[1.0-3.0]$ & $3.8[2.0-6.0]$ & $0.84[0.7-1.0]$ & $<0.0001$ & $<0.0001$ & $<0.0001$ \\
\hline $\begin{array}{l}\text { Number of organs involved; mean (median), } \\
\text { [IQR] }\end{array}$ & $3.27(3)[2-4]$ & $2.4(2)[2-3]$ & $3.82(4)[3-5]$ & $<0.0001$ & $<0.01$ & $<0.0001$ \\
\hline Observation time (month); median, [IQR] & $\begin{array}{l}61.9 \\
{[24.3-104.3]}\end{array}$ & $\begin{array}{l}27.4 \\
\quad[10.5-48.8]\end{array}$ & $\begin{array}{l}68 \\
\quad[35.3-113 .- \\
1]\end{array}$ & $<0.0001$ & NS & $<0.0001$ \\
\hline Patients with relapse $n(\%)$ & $243(58.3)$ & $27(25.5)$ & $70(68.6)$ & $<0.00001$ & NS & $<0.00001$ \\
\hline Relapse rate ${ }^{\&}$ & $18.0 \pm 0.8$ & $12.0 \pm 1.9$ & $25.2 \pm 1.9$ & $<0.05$ & $<0.05$ & $<0.01$ \\
\hline Time to relapse $^{6}$ (month); median, [IQR] & $16[8-31]$ & $17[9-45]$ & $11[4.5-19]$ & NS & $<0.001$ & $<0.05$ \\
\hline CTX $\operatorname{median}^{7}(\mathrm{~g}) ;[\mathrm{IQR}]$ & $9[5.35-16]$ & $5[2-7]$ & $6[5.7-8.36]$ & $<0.0001$ & NS & NS \\
\hline Permanent renal replacement therapy $n(\%)$ & $55(13.2)$ & $36(34)$ & 0 & $<0.0001$ & $<0.0001$ & $<0.0001$ \\
\hline Temporary renal replacement therapy $n(\%)$ & $32(7.7)$ & $11(10.4)$ & 0 & NS & $<0.01$ & $<0.001$ \\
\hline
\end{tabular}

${ }^{\&}$ Relapse rate $=$ number of relapses per 100 patients per 1 year

${ }^{1}$ Positive by either IIF or ELISA, or both

${ }^{2}$ Positive for either cANCA by IIF or anti-PR3 by ELISA, or both

${ }^{3}$ Positive for either pANCA by IIF or anti-MPO by ELISA, or both

${ }^{4}$ Time from reported onset of symptoms to diagnosis

${ }^{5}$ Creatinine max-maximum creatinine concentration ever

${ }^{6}$ Time to relapse $=$ time in months between diagnosis and the first disease relapse

${ }^{7}$ Cumulative dose of cyclophosphamide (CTX)

$E N T$, ear, nose, throat; $C N S$, central nervous system; GI, gastrointestinal tract; IQR, interquartile range, $C T X$, cyclophosphamide

requiring dialysis $(\mathrm{M} / \mathrm{F}, 53 / 34 ; p<0.05)$. Dialyzed GPA patients also showed significantly higher serum CRP concentration at baseline (median $54 \mathrm{mg} / \mathrm{l}$ vs $32 \mathrm{mg} / \mathrm{l} ; p<0.05$ ).

In the subgroup of MPA patients defined by dialysis status, no differences in their demographic characteristics were observed. The only clinical difference was GI tract involvement which was more common in dialyzed patients $(19.1 \%$ vs $5.1 \%, p<0.05$ ) (Table 3).

Patients who died, both in GPA and MPA group experienced disease relapses more often. For this reason, we decided 
Table 2 Factors related to death in AAV patients

\begin{tabular}{|c|c|c|c|c|}
\hline & \multicolumn{2}{|l|}{ GPA } & \multicolumn{2}{|l|}{ MPA } \\
\hline & Death cases & Alive patients & Death cases & Alive patients \\
\hline No. of patients & 42 & 375 & 13 & 93 \\
\hline Age (years); median & 55.5 & 51.1 & 71.1 & $62.9^{+}$ \\
\hline Male/female & $26 / 16$ & $184 / 191$ & $6 / 7$ & $48 / 45$ \\
\hline Time to diagnosis ${ }^{1}$ (month); median, $[\mathrm{IQR}]$ & $6.0[4.0-14]$ & $5.9[3.0-13.9]$ & $4.0[1.8-7.4]$ & $5.3[2.0-12.0]$ \\
\hline Diagnosis to 1. Relapse (month); median, [IQR] & $12[7.8-24]$ & $18[8.5-33.5]$ & $26.5[14.25-39] n=5$ & $17[9-45]$ \\
\hline Average observation time (month) & 66.2 & $72.6^{+}$ & 20.55 & 36.2 \\
\hline Number of organs involved; mean (median), [IQR] & $3.73(4)[3-4]$ & $3.22(3)[2-4]^{*}$ & $3.15(3)[2-4]$ & $2.3(2)[2-3]^{*}$ \\
\hline Permanent renal replacement therapy; $n(\%)$ & $15(35.7)$ & $40(10.7)^{\#}$ & $8(61.5)$ & $28(30.1)^{\#}$ \\
\hline Temporary renal replacement therapy; $n(\%)$ & $4(9.5)$ & $28(7.5)$ & $1(7.7)$ & $10(10.7)$ \\
\hline Patients with at least one relapse; $n(\%)$ & $28(66.7)$ & $214(57.1)^{\#}$ & $5(38.4)$ & $22(23.7)^{\#}$ \\
\hline Relapse rate ${ }^{\&}$ & $26.4 \pm 3.4$ & $19.2 \pm 1.0$ & $26.4 \pm 10.8$ & $12.0 \pm 2.4$ \\
\hline \multicolumn{5}{|l|}{ Organ involvement } \\
\hline General; $n(\%)$ & $37(88.1)$ & $318(84.8)$ & $12(92.3)$ & $71(76.3)$ \\
\hline Skin; $n(\%)$ & $17(40.5)$ & $123(32.8)$ & $6(46.1)$ & $18(19.3)^{*}$ \\
\hline Eye; $n(\%)$ & $7(16.7)$ & $107(28.5)$ & $0(0)$ & $4(4.3)$ \\
\hline $\mathrm{ENT} ; n(\%)$ & $31(73.8)$ & $284(75.7)$ & $5(38.4)$ & $13(13.9)^{*}$ \\
\hline Respiratory; $n(\%)$ & $38(90.5)$ & $277(73.9)^{*}$ & $9(69.2)$ & $51(54.8)$ \\
\hline Heart; $n(\%)$ & $8(19.1)$ & $47(12.5)$ & $0(0)$ & $11(11.8)$ \\
\hline $\mathrm{GI} ; n(\%)$ & $6(14.3)$ & $40(10.7)$ & $3(23.1)$ & $9(9.7)$ \\
\hline Renal; $n(\%)$ & $32(76.2)$ & $220(58.7)^{*}$ & $12(92.3)$ & $89(95.6)$ \\
\hline Urinary; $n(\%)$ & $1(2.4)$ & $7(1.7)$ & 0 & $3(3.2)$ \\
\hline $\mathrm{CNS} ; n(\%)$ & $6(14.3)$ & $39(10.4)$ & $2(15.4)$ & $0(0)$ \\
\hline Peripheral nerves; $n(\%)$ & $11(26.1)$ & $64(17.1)$ & $4(30.8)$ & $16(17.2)$ \\
\hline Creatinine $\max ^{2} ;(\mathrm{mg} / \mathrm{dl}) ;$ median $[\mathrm{IQR}]$ & $3[1.0-6.7]$ & $1.03[1.0-2.8]^{*}$ & $5[4.0-6.2]$ & $3[2.0-6.0]^{+}$ \\
\hline CRP at baseline (mg/l); median, [IQR] & $64[22.5-130]$ & $33.2[8.9-92.8]$ & $49[33.3-116.5]$ & $30.5[13.0-81.0]$ \\
\hline CTX $(\mathrm{g})^{3} ;$ median, [IQR] & $7.1[3.0-13.5]$ & $9[6-16.2]$ & $1.5[1.0-3.25]$ & $5.1[2.4-8.0]^{+}$ \\
\hline Smoker/previous smoker/no/missing $(n)^{4}$ & $3 / 7 / 10 / 22$ & $22 / 65 / 173 / 92$ & $0 / 1 / 5 / 7$ & $5 / 36 / 34 / 17$ \\
\hline
\end{tabular}

${ }^{*} p<0.05 ;{ }^{+} p<0.01 ;{ }^{\#} p<0.001$

${ }^{\&}$ Relapse rate $=$ number of relapses per 100 patient per 1 year

${ }^{1}$ Time from reported onset of symptoms to diagnosis

${ }^{2}$ Maximal serum creatinine concentration ever. Values after initiation of renal replacement therapy are excluded

${ }^{3}$ Cumulative dose of cyclophosphamide (CTX)

${ }^{4}$ Statistical analysis compared smokers and previous smokers vs no (subjects who never smoked)

$E N T$, ear, nose, throat; $C N S$, central nervous system; GI, gastrointestinal tract, $I Q R$, interquartile range, $C T X$, cyclophosphamide, numbers in italic significant statistical difference between groups

to analyze further possible factors influencing relapse rate (Suppl. Tab. S4).

In the GPA group (during an average observation time of 49.1 months), 175 patients (42\%) never experienced a relapse, while $58 \%$ experienced at least one relapse (average observation time 96.7 months). In MPA patients, only 27 patients $(34 \%)$ relapsed (average observation time in relapsing group - 64.2 months). Observation time in both subgroups differed, but after adjustment for the duration of the disease, their relapse rate was very similar (Suppl. Tab. S4).

Relapsing GPA patients were younger than the subgroup without any relapse. They also showed increased ENT and eye involvement ( $81 \%$ vs $68 \% ; p<0.01$ and $31.8 \%$ vs $21.1 \%$; $p<0.01)$ and low rate of GI tract involvement $(5.8 \%$ vs $14.1 \% ; p<0.01)$.

Relapsing MPA subgroup did not differ by age from the non-relapsing ones. Clinical differences between these groups resembled, although less significantly, GPA subjects.

As we observed, an increased participation of men among those GPA patients who died and those requiring RRT (Table 2 and Suppl. Tab. S4), we decided to see into possible differences in the disease course and organ involvement between male and female AAV subjects. 
Table 3 Factors related to renal replacement therapy in AAV patients

\begin{tabular}{|c|c|c|c|c|}
\hline & \multicolumn{2}{|l|}{ GPA } & \multicolumn{2}{|l|}{ MPA } \\
\hline & No RRT & Any RRT & No RRT & Any RRT \\
\hline No. of patients & 330 & 87 & 59 & 47 \\
\hline Age (years); median & 50.1 & $56.5^{\#}$ & 60.5 & 64.7 \\
\hline Male/female & $157 / 173$ & $53 / 34^{*}$ & $32 / 27$ & $22 / 25$ \\
\hline Time to diagnosis ${ }^{1}$ (month); median [IQR] & $6.0[3.0-14.1]$ & $4.95[2.0-10]$ & $3.9[2.0-13.6]$ & $5.8[2-10.5]$ \\
\hline Diagnosis to 1. Relapse (month); median, [IQR] & $17.5[8-30]$ & $15[10-33]$ & $27[13-48]$ & $17[7.5-28]$ \\
\hline Average observation time (month) & 75.1 & 82.8 & 39.9 & 31.7 \\
\hline Number of organs involved; mean (median) [IQR] & $3.25(3)[2-4]$ & $3.35(3)[2-4]$ & $2.37(2)[2,3]$ & $2.44(2)[2,3]$ \\
\hline Permanent renal replacement therapy $n(\%)$ & NA & $55(63.2)$ & NA & $36(76.6)$ \\
\hline Temporary renal replacement therapy $n(\%)$ & NA & $32(36.8)$ & NA & $11(23.4)$ \\
\hline Death; $n(\%)$ & $23(7.0)$ & $19(21.8)^{\#}$ & $4(6.8)$ & $9(19.1)$ \\
\hline Patients with relapse $n(\%)$ & $196(59.4)$ & $46(52.9)$ & $15(25.4)$ & $12(25.5)$ \\
\hline Relapse rate ${ }^{\&}$ & $19.2 \pm 1.0$ & $15.0 \pm 1.6$ & $10.8 \pm 2.4$ & $14.4 \pm 4.8$ \\
\hline \multicolumn{5}{|l|}{ Organ involvement } \\
\hline General $n(\%)$ & $274(83.0)$ & $81(93.1)$ & $45(76.3)$ & $38(80.8)$ \\
\hline Skin $n(\%)$ & $112(33.9)$ & $28(32.2)$ & $16(27.1)$ & $8(17)$ \\
\hline Eye $n(\%)$ & $100(30.3)$ & $14(16.1)$ & $4(6.8)$ & $0(0)$ \\
\hline ENT $n(\%)$ & $271(82.1)$ & $44(50.6)^{\#}$ & $9(15.3)$ & $9(19.1)$ \\
\hline Respiratory $n(\%)$ & $245(74.2)$ & $70(80.5)$ & $33(55.9)$ & $27(57.5)$ \\
\hline Heart $n(\%)$ & $43(13.0)$ & $12(13.8)$ & $4(6.8)$ & $7(14.9)$ \\
\hline GI $n(\%)$ & $32(9.7)$ & $14(16.1)$ & $3(5.1)$ & $9(19.1)^{*}$ \\
\hline Renal $n(\%)$ & $165(50)$ & $87(100)$ & $55(93.2)$ & $47(100)$ \\
\hline Urinary $n(\%)$ & $5(1.5)$ & $3(3.4)$ & $2(3.4)$ & $1(2.1)$ \\
\hline CNS $n(\%)$ & $37(11.2)$ & $8(9.2)$ & $0(0)$ & $2(4.2)$ \\
\hline Peripheral nerves $n(\%)$ & $64(19.4)$ & $11(12.6)$ & $14(23.7)$ & $6(12.8)$ \\
\hline Creatinine $\max ^{2} ;(\mathrm{mg} / \mathrm{dl}) ;$ median, [IQR] & $1[1.0-1.7]$ & $6.0[4.5-8.1]^{\#}$ & $2.07[1.2-3.0]$ & $6.1[5.0-7.75]^{\#}$ \\
\hline $\mathrm{CRP}$ at baseline (mg/l); median, [IQR] & $32[8.7-88.7]$ & $54[18-138]^{*}$ & $30[16-76.6]$ & $34.8[11.1-103.5]$ \\
\hline $\mathrm{CTX}(\mathrm{g})^{3} ;$ median, $[\mathrm{IQR}]$ & $9[6-17]$ & $7.2[4-15]$ & $6.3[3.5-9.6]$ & $2.4[1.3-5.0]^{\#}$ \\
\hline Smoker/previous smoker/no/missing $(n)^{4}$ & 19/52/160/77 & $6 / 20 / 23 / 36^{+}$ & $4 / 18 / 25 / 11$ & $1 / 19 / 14 / 13$ \\
\hline
\end{tabular}

See Table 2 for legend

Surprisingly, in the GPA group, male patients compared to women showed significantly more often respiratory $(81.4 \%$ vs $69.6 \% ; p<0.01)$ and renal $(70.9 \%$ vs $49.8 \% ; p<0.00001)$ involvement, but suffered from ENT symptoms slightly less frequently $(71.4 \%$ vs $79.7 \% ; p<0.05)$. Higher rate of renal and lung involvement in men may have facilitated final diagnosis exemplified by a significantly shorter time to diagnosis in male GPA patients (median 4.9 months vs 7.0 months; $p<0.01$; see Tab.4). In contrast, men and women in the MPA (Table 4) and EGPA (data not shown) groups showed only single, isolated differences.

To identify factors independently related to death and the requirement for PRRT in AAV patients, we additionally performed logistic regression analysis (Table 5). For analysis, we have chosen parameters significantly different between groups (death vs alive; any RRT vs no RRT) in previous analyses $(p<0.05)$. Analysis included whole AAV group (here specificity of ANCA antibodies was also added), GPA, and MPA. EGPA group was not included as there was only one death among EGPA patients, and no need for renal replacement therapy was reported. In the whole AAV group, independent risk factors for death in univariate analysis were PRRT, kidney involvement, age $>65$ years, and the presence of c/PR3 ANCA, while in multivariate analysis, it included PRRT, respiratory involvement, age $>65$ years, and the presence of c/PR3 ANCA. As PRRT risk factors univariate analysis identified: relapsing disease, age $>65$, p/MPO ANCA, and ENT involvement as a factor negatively associated with PRRT, whereas multivariate analysis confirmed only a role of ENT involvement and the presence of p/MPO ANCA, and added as a significant, although weak risk factor, the presence of c/PR3 ANCA.

In GPA patients, univariate analysis identified the following risk factors related to death: PRRT and lower 
Table 4 Demographic characteristics and comparison of organ involvement and laboratory parameters of GPA and MPA patients (male vs female)

\begin{tabular}{|c|c|c|c|c|}
\hline & \multicolumn{2}{|l|}{ GPA } & \multicolumn{2}{|l|}{ MPA } \\
\hline & Male & Female & Male & Female \\
\hline No. of patients & 210 & 207 & 54 & 52 \\
\hline Age (years); median & 51.0 & 52.6 & 60.7 & 66.1 \\
\hline Time to diagnosis ${ }^{1}$ (month); median, [IQR $]$ & $4.9[2.24-12.0]$ & $7.0[3.0-20.4]^{+}$ & $3.9[2-9.2]$ & $6.9[2-12.8]$ \\
\hline Diagnosis to 1. Relapse (month); median [IQR] & $14[7.5-31.5]$ & $21.5[10-30]$ & 16 [9-45] & 18.5 [13.75-39] \\
\hline Average observation time (month); (median), [IQR] & $\begin{array}{l}77.4(59.0) \\
\quad[23.4-106.1]\end{array}$ & $\begin{array}{l}75.9(62.4) \\
\quad[22.3-71.5]\end{array}$ & $\begin{array}{l}37.7 \\
\quad(27.9)[10.8-52 .- \\
\quad 7]\end{array}$ & $\begin{array}{l}34.6(23.9) \\
\quad[8.5-43.6]\end{array}$ \\
\hline Number of organs involved; mean (median) [IQR] & $3.37(3)[2-4]$ & $3.17(3)[2-4]$ & $2.62(2)[2-3.75]$ & $2.17(2)[1-3]$ \\
\hline Permanent renal replacement therapy $n(\%)$ & $32(15.2)$ & $23(11.1)$ & $18(33.3)$ & $18(34.6)$ \\
\hline Temporary renal replacement therapy $n(\%)$ & $21(10)$ & $11(5.3)$ & $4(7.4)$ & $7(13.5)$ \\
\hline Death $n(\%)$ & $26(12.4)$ & $16(7.7)$ & $6(11.1)$ & $7(13.5)$ \\
\hline Patients with relapse $n(\%)$ & $130(61.9)$ & $112(54.1)$ & $15(27.7)$ & $12(23.1)$ \\
\hline Relapse rate ${ }^{\&}$ & $19.2 \pm 1.2$ & $16.8 \pm 1.2$ & $13.2 \pm 2.4$ & $10.8 \pm 2.4$ \\
\hline \multicolumn{5}{|l|}{ Organ involvement } \\
\hline General $n(\%)$ & $180(85.7)$ & $175(84.5)$ & $39(72.2)$ & $44(84.6)$ \\
\hline Skin $n(\%)$ & $74(35.2)$ & $66(31.9)$ & $12(22.2)$ & $12(23.1)$ \\
\hline Eye $n(\%)$ & $46(21.9)$ & $68(32.8)$ & $2(3.7)$ & $2(3.9)$ \\
\hline ENT $n(\%)$ & $150(71.4)$ & $165(79.7)^{*}$ & $11(20.4)$ & $7(13.5)$ \\
\hline Respiratory $n(\%)$ & $171(81.4)$ & $144(69.6)^{+}$ & $34(62.9)$ & $26(50.0)$ \\
\hline Heart $n(\%)$ & $31(14.8)$ & $24(11.6)$ & $8(14.8)$ & $3(5.8)$ \\
\hline GI $n(\%)$ & $21(10.0)$ & $25(12.1)$ & $8(14.8)$ & $4(7.7)$ \\
\hline Renal $n(\%)$ & $149(70.9)$ & $103(49.8)^{\#}$ & $51(94.4)$ & $50(96.2)$ \\
\hline Urinary $n(\%)$ & $4(1.9)$ & $4(1.9)$ & $2(3.7)$ & $1(1.9)$ \\
\hline $\mathrm{CNS} n(\%)$ & $22(10.5)$ & $23(11.1)$ & $2(3.7)$ & $0(0)$ \\
\hline Peripheral nerves $n(\%)$ & $40(19.1)$ & $35(16.9)$ & $12(22.2)$ & $8(15.4)$ \\
\hline Creatinine $\max ^{2}(\mathrm{mg} / \mathrm{dl}) ;$ median, [IQR] & $1.6[1.0-4.52]$ & $1.0[0.9-2.0]^{\#}$ & $3.0[1.85-5.37]$ & $4.3[2.0-6.14]$ \\
\hline $\begin{array}{l}\text { Subjects with renal involvement creatinine max }(\mathrm{mg} / \mathrm{dl}) \text {; median, } \\
\text { [IQR] }\end{array}$ & $2.5[1.3-5.7]$ & $2.0[1-4.0]^{*}$ & & \\
\hline $\begin{array}{l}\text { Subjects without renal involvement creatinine } \max (\mathrm{mg} / \mathrm{dl}) ; \\
\text { median, }[\mathrm{IQR}]\end{array}$ & $1.0[0.99-1]$ & $1.0[0.8-1]$ & & \\
\hline $\mathrm{CRP}$ at baseline (mg/l); median, [IQR] & $46[10-115.5]$ & $31[8-89]$ & $35[19-86]$ & $32[10-78]$ \\
\hline $\operatorname{CTX}(\mathrm{g})^{3} ;$ median, $[\mathrm{IQR}]$ & $9[5-20]$ & $8[5.7-14.5]$ & $6[2-9.25]$ & $3.5[1.5-7]^{*}$ \\
\hline Smoker/previous smoker/no/missing $(n)^{4}$ & $20 / 48 / 69 / 61$ & $5 / 24 / 114 / 52^{\#}$ & $5 / 24 / 11 / 13$ & $0 / 23 / 29 / 0^{+}$ \\
\hline
\end{tabular}

See Table 2 for legend

respiratory tract involvement. Multivariate analyses added elder age at diagnosis ( $>65$ years). Analysis of risk factors for PRRT identified ENT involvement as a factor negatively associated with risk of dialysis by both univariate and multivariate analysis. In addition, multivariate analyses identified multiorgan disease as directly and independently related to PRRT $(p<0.01)$.

In MPA patients, factors related to death by univariate analysis include PRRT, skin and ENT involvement, and the age > 65 years at diagnosis. Multivariate logistic regression analyses confirmed significance of all three factors. In the MPA group, among factors analyzed, none was identified by regression analysis as related to PRRT.

\section{Discussion}

POLVAS registry is the first large database of AAV patients in Poland. Data were obtained from tertiary reference centers representing several specialties to cover a spectrum of different possible symptomatology at presentation and during the course of the disease [17]. In general, major demographic characteristics and patterns of organ involvement in our ethnically homogenous Caucasian population were consistent with already published major AAV series from the UK, French, and Spanish cohorts [8, 9], [18-20]. Frequency of AAV was also typical for other European countries. Male to female ratio was 1:1 for GPA and MPA, and 1:2 in for EGPA. 


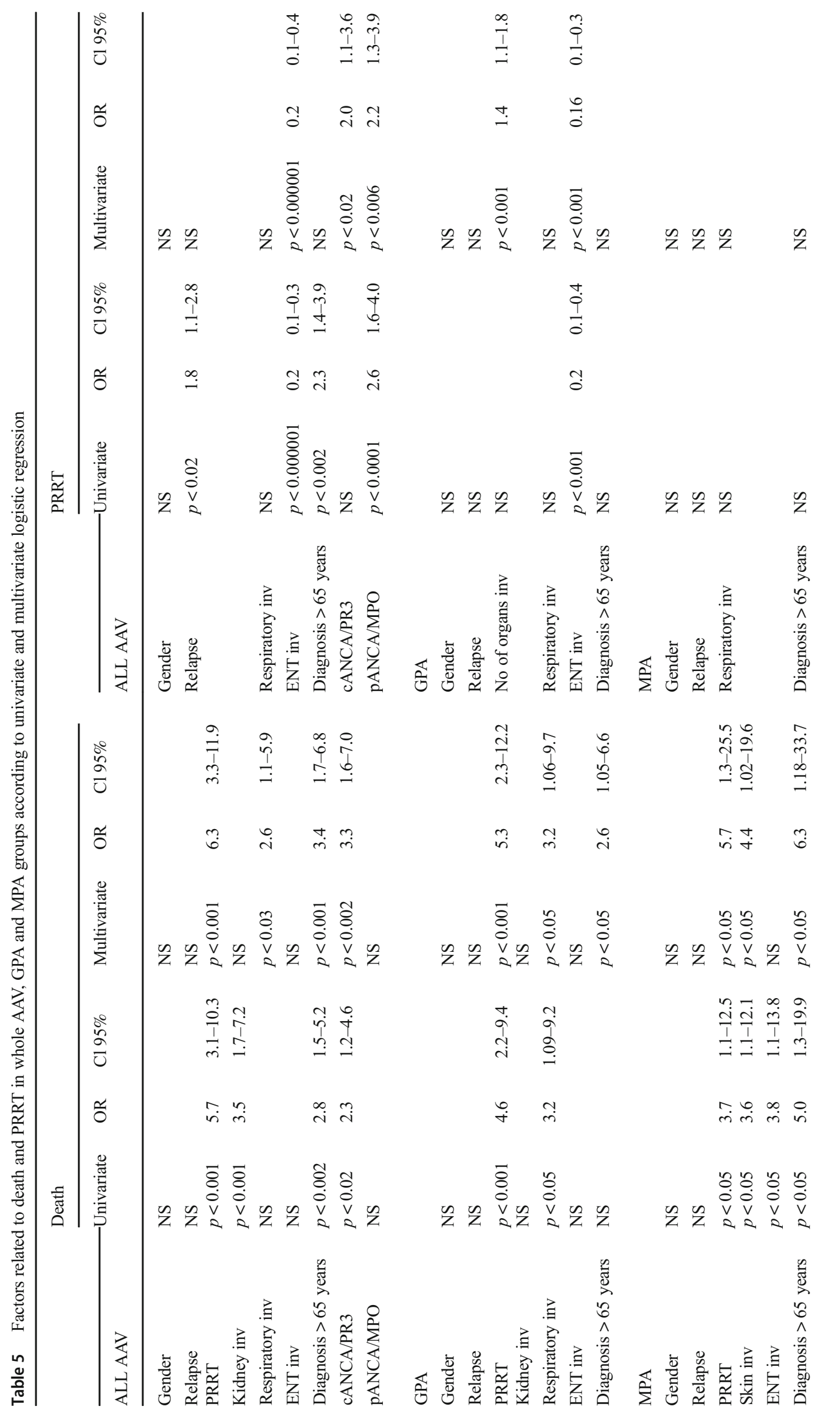


This corresponds to other recently published Polish report [21] and, unexpectedly, to data obtained in the Japanese population [22], but differs from the results published by French Vasculitis Study Group and from Spain [19, 20]. As the proportion of anti-MPO positive and anti-MPO negative EGPA patients in our cohort was similar to the one observed in other European countries [2], higher proportion of women in our registry may represent a true difference between Central and South-Western Europe. Ethnic and geographic differences in epidemiology and clinical presentation of AAV have been already described in other populations throughout the world $[8,9],[10,23]$.

At this point, it is important to note that there were important differences within EGPA group depending on their ANCA status. The presence of anti-MPO ANCA in EGPA not only modified the age at disease diagnosis but also its clinical manifestations. This is in accordance with other clinical reports describing ANCA-positive EGPA patients [11, 19]. EGPA may also pose some problems with definitions. For example, in our cohort, the highest frequency of relapses was surprisingly observed in EGPA patients. However, it might rather reflect asthma exacerbations than true vasculitic relapse [24]. It is than well substantiated to analyze EGPA patients separately from other AAV subjects [25] with ANCA status as an important differentiating factor [26]. Of interest, almost $3 \%$ of our GPA patients in whom differential blood smear was available showed increased blood eosinophil count as defined by Lanham [27].

Usually, AAVs are characterized by a multisystem involvement. Accordingly, in Polish patients, the highest number of organs involved was found in the EGPA group (median 3.84 organs), followed by GPA (3.27), and finally MPA (2.4), which was consistent with recently published data [18]. Differences between clinical presentation and laboratory findings in GPA and MPA exist, but they overlap making differential diagnosis between these two clinical entities often difficult. As it could be expected, also in POLVAS patients, $14 \%$ of pANCA/anti-MPO positive subjects were diagnosed with GPA while 5\% of MPA (or EGPA) patients showed cANCA/ anti-PR3 positivity.

In recent decades, a significant progress has been made in effective treatment of AAV, an often life-threatening multiorgan systemic diseases. The 5 -year survival rate is now estimated by some authors at $94.5 \%$ [28]. Others report the 5-year survival in GPA ranging between 73 and $83 \%$ and in MPA, between 45 and $85 \%$ [23, 29]. Despite a significant improvement in survival noted between the last decades of the twentieth century as compared to recent years [28,29], mortality in $\mathrm{AAV}$ is still about three times higher than in the general population $[28,29]$. For this reason, it seems of utmost importance to identify factors related to the poor outcome in AAV patients.
In POLVAS AAV patients, predictors of death identified by logistic regression analysis slightly differed depending on the AAV phenotype. Independent risk factors for death in both GPA and MPA patients included age at diagnosis over 65 years, chronic renal replacement therapy, and lower respiratory tract involvement. In addition, in MPA, skin involvement was also independently associated with mortality which might represent a more generalized disease. Overall, our results concur with the findings from other AAV cohorts $[9,20,30]$. However, a word of caution is required while comparing different reports, as sometimes terms defining kidney disease differed, including "any renal involvement" or "increased serum creatinine concentration" $[9,20,31]$. To clarify this issue, we performed additional analyses applying those different definitions of renal involvement. Only PRRT and maximal creatinine concentration ever (data not shown) remained independently associated with death in our AAV cohort. The general term "kidney involvement" did not reach statistical significance in these analyses $(p=$ 0.064).

As PRRT was independently associated with death in both GPA and MPA, we further attempted to identify risk factors associated with PRRT. Again, the factors slightly differed depending on the AAV phenotype. In GPA, a number of organs involved was positively associated with PRRT while ENT involvement seemed to represent a protective factor. This latter finding indicates that, in fact, limited form of GPA is characterized by a more benign disease course [32]. Logistic regression analysis of the whole AAV group with the addition of ANCA specificity identified the presence of c/PR3 ANCA (for death) and of both, c/PR3 and p/MPO ANCA for PRRT. We did not analyzed risk factors for relapse (as an outcome measure) in our patients as we identified time from diagnosis (the length of retrospective observation) as the main factor associated with the first and further relapse episodes.

Analysis of outcome in GPA patients suggested that there were more men among those patients who died, although, in our hands, this difference was not statistically significant. Men were more prevalent among chronically dialyzed subjects with GPA (see Table 4.). This corroborated with a higher rate of kidney and lower respiratory tract involvement and lower rate of ENT involvement - all factors identified as independently related to poor prognosis. An increase in mortality during the first 2 years of AAV was already observed among Swedish AAV patients [33]. Interestingly, delay from the first symptoms of disease to diagnosis was in males shorter than in females. Thus, it was not an unduly delayed diagnosis but rather a truly more severe disease course from the beginning which characterizes men with GPA. Indeed, there are some reports suggesting that, at least in terms of kidney involvement, this might be the case [34-36]. These results also raise 
the question about the most appropriate treatment regimen and monitoring in men with GPA.

The already mentioned, higher mortality observed during the first 2 years from AAV diagnosis in Swedish men changed to a higher mortality in women but only after about 8 years of observation [33]. Longer times of observation of our cohort may be needed to confirm those findings. In POLVAS registry, median observation time slightly exceeded 5 years in GPA and EGPA subjects and about 2.5 years in MPA patients.

Less than $5 \%$ of patients in our cohort was recruited between 1990 and 2000. The main reasons were possibly: the availability of ANCA tests and less well-defined entities inside AAVgroup. This is nicely reflected in the recent report from French Vasculitis Study Group showing a dramatic change in proportion of patients diagnosed with polyarteritis nodosa and GPA in the last decades of the twentieth century as compared with the years after 2000 [28].

Properly planned prospective clinical trials are needed to offer definite answers to the questions raised by our data.

\section{Summary}

In summary, this is the first multicenter retrospective study of Polish patients with AAV. Demographic characteristics and disease manifestation of patients in POLVAS registry are similar to those from other European countries. Following independent risk factors related to death were identified in both GPA and MPA: age at diagnosis $>65$ years and PRRT. In addition, risk factors for death in GPA included lower respiratory tract involvement and in MPA, skin involvement. We also identified more frequent kidney and lower respiratory tract involvement in male patients with GPA. This observation requires confirmation in prospective clinical trials, and maybe a change in the therapeutic approach in male patients.

Funding This work was supported by Jagiellonian University Medical College grant no. K/ZDS/007903.

\section{Compliance with ethical standards}

\section{Disclosures None.}

Open Access This article is distributed under the terms of the Creative Commons Attribution 4.0 International License (http:// creativecommons.org/licenses/by/4.0/), which permits unrestricted use, distribution, and reproduction in any medium, provided you give appropriate credit to the original author(s) and the source, provide a link to the Creative Commons license, and indicate if changes were made.

\section{References}

1. Seo P, Stone JH (2004) The antineutrophil cytoplasmic antibodyassociated vasculitides. Am J Med 117:39-50. https://doi.org/10. 1016/j.amjmed.2004.02.030
2. Millet A, Pederzoli-Ribeil M, Guillevin L, Witko-Sarsat V, Mouthon L (2013) Antineutrophil cytoplasmic antibodyassociated vasculitides: is it time to split up the group? Ann Rheum Dis 72:1273-1279. https://doi.org/10.1136/annrheumdis2013-203255

3. Jennette JC (2013) Overview of the 2012 revised International Chapel Hill Consensus Conference nomenclature of vasculitides. Clin Exp Nephrol 17:603-606. https://doi.org/10.1007/s10157013-0869-6

4. Tan JA, Dehghan N, Chen W, Xie H, Esdaile JM, Avina-Zubieta JA (2017) Mortality in ANCA-associated vasculitis: ameta-analysis of observational studies. Ann Rheum Dis 76:1566-1574. https://doi. org/10.1136/annrheumdis-2016-210942

5. Seror R, Pagnoux C, Ruivard M, Landru I, Wahl D, Riviere S, Aussant S, Mahr A, Cohen P, Mouthon L, Guillevin L, for the French Vasculitis Study Group (2010) Treatment strategies and outcome of induction-refractory Wegener's granulomatosis or microscopic polyangiitis: analysis of 32 patients with first-line induction-refractory disease in the WEGENT trial. Ann Rheum Dis 69: 2125-2130. https://doi.org/10.1136/ard.2010.131953

6. Walsh M, Flossmann O, Berden A, Westman K, Höglund P, Stegeman C, Jayne D, European Vasculitis Study Group (2012) Risk factors for relapse of antineutrophil cytoplasmic antibodyassociated vasculitis. Arthritis Rheum 64:542-548. https://doi.org/ 10.1002/art.33361

7. Mahr A, Katsahian S, Varet H, Guillevin L, Hagen EC, Höglund P, Merkel PA, Pagnoux C, Rasmussen N, Westman K, Jayne DRW, for the French Vasculitis Study Group (FVSG) and the European Vasculitis Society (EUVAS) (2013) Revisiting the classification of clinical phenotypes of anti-neutrophil cytoplasmic antibodyassociated vasculitis: a cluster analysis. Ann Rheum Dis 72:10031010. https://doi.org/10.1136/annrheumdis-2012-201750

8. Furuta S, Chaudhry AN, Hamano Y, Fujimoto S, Nagafuchi H, Makino H, Matsuo S, Ozaki S, Endo T, Muso E, Ito C, Kusano E, Yamagata M, Ikeda K, Kashiwakuma D, Iwamoto I, Westman K, Jayne D (2014) Comparison of phenotype and outcome in microscopic polyangiitis between Europe and Japan. J Rheumatol 41: 325-333 https://doi.org/10.3899/jrheum.130602

9. Furuta S, Chaudhry AN, Arimura Y, Dobashi H, Fujimoto S, Homma S, Rasmussen N, Jayne DR (2017) Comparison of the phenotype and outcome of granulomatosis with polyangiitis between UK and Japanese cohorts. J Rheumatol 44:216-222 https:// doi.org/10.3899/jrheum.160005

10. Pearce FA, Craven A, Merkel PA, Luqmani RA, Watts RA (2017) Global ethnic and geographic differences in the clinical presentations of anti-neutrophil cytoplasm antibody-associated vasculitis. Rheumatol Oxf Engl 56:1962-1969 https://doi.org/10.1093/ rheumatology/kex293

11. Sokolowska BM, Szczeklik WK, Wludarczyk AA et al (2014) ANCA-positive and ANCA-negative phenotypes of eosinophilic granulomatosis with polyangiitis (EGPA): outcome and long-term follow-up of 50 patients from a single Polish center. Clin Exp Rheumatol 32:S41-S47

12. Wisłowska M, Gozdowska J (2008) Original paper $<$ BR $>$ Wegener's granulomatosis - observation of cases. Reumatologia/Rheumatology 46:68-71

13. Padjas A, Sznajd J, Szczeklik W et al (2014) Rare disease registries: an initiative to establish vasculitis registry in Poland. Pol Arch Med Wewn 124:143-144

14. Musiał J, Wójcik K (2017) Polish Vasculitis Registry: POLVAS. Pol Arch Intern Med 127:71-72. https://doi.org/10.20452/pamw. 3920

15. Bajema IM, Bruijn JA, Casian A, Cid MC, Csernok E, van Daalen E, Harper L, Hauser T, Little MA, Luqmani RA, Mahr A, Ponte C, Salama A, Segelmark M, Suzuki K, Sznajd J, Teng YKO, Vaglio A, Westman K, Jayne D (2017) The European Vasculitis Society 2016 
Meeting Report. Kidney Int Rep 2:1018-1031. https://doi.org/10. 1016/j.ekir.2017.09.008

16. Mukhtyar C, Lee R, Brown D, Carruthers D, Dasgupta B, Dubey S, Flossmann O, Hall C, Hollywood J, Jayne D, Jones R, Lanyon P, Muir A, Scott D, Young L, Luqmani RA (2009) Modification and validation of the Birmingham Vasculitis Activity Score (version 3). Ann Rheum Dis 68:1827-1832 https://doi.org/10.1136/ard.2008. 101279

17. McNicholas BA, Griffin TP, Donnellan S et al (2016) ANCAassociated vasculitis: a comparison of cases presenting to nephrology and rheumatology services. QJM Int J Med 109:803-809. https://doi.org/10.1093/qjmed/hcw100

18. Guillevin L, Pagnoux C, Seror R, Mahr A, Mouthon L, Toumelin PL (2011) The five-factor score revisited: assessment of prognoses of systemic necrotizing vasculitides based on the French Vasculitis Study Group (FVSG) cohort. Medicine (Baltimore) 90:19-27. https://doi.org/10.1097/MD.0b013e318205a4c6

19. Comarmond C, Pagnoux C, Khellaf M, Cordier JF, Hamidou M, Viallard JF, Maurier F, Jouneau S, Bienvenu B, Puéchal X, Aumaître O, Guenno GL, Quellec AL, Cevallos R, Fain O, Godeau B, Seror R, Dunogué B, Mahr A, Guilpain P, Cohen P, Aouba A, Mouthon L, Guillevin L, for the French Vasculitis Study Group (2013) Eosinophilic granulomatosis with polyangiitis (Churg-Strauss): clinical characteristics and long-term followup of the 383 patients enrolled in the French Vasculitis Study Group cohort. Arthritis Rheum 65:270-281 https://doi.org/10.1002/art. 37721

20. Solans-Laqué R, Fraile G, Rodriguez-Carballeira M, Caminal L, Castillo MJ, Martínez-Valle F, Sáez L, Rios JJ, Solanich X, Oristrell J, Pasquau F, Fonseca E, Zamora M, Callejas JL, Frutos B, Abdilla M, Fanlo P, García-Sánchez I, López-Dupla M, Sopeña B, Pérez-Iglesias A, Bosch JA (2017) Clinical characteristics and outcome of Spanish patients with ANCA-associated vasculitides: impact of the vasculitis type, ANCA specificity, and treatment on mortality and morbidity. Medicine (Baltimore) 96:e6083. https:// doi.org/10.1097/MD.0000000000006083

21. Kanecki K, Nitsch-Osuch A, Gorynski P et al (2017) Hospital morbidity database for epidemiological studies on Churg-Strauss syndrome. Adv Exp Med Biol 980:19-25. https://doi.org/10.1007/ 5584_2017_8

22. Sada K, Yamamura M, Harigai M, Fujii T, Dobashi H, Takasaki Y, Ito S, Yamada H, Wada T, Hirahashi J, Arimura Y, Makino H, the Research Committee on Intractable Vasculitides, the Ministry of Health, Labour and Welfare of Japan (2014) Classification and characteristics of Japanese patients with antineutrophil cytoplasmic antibody-associated vasculitis in a nationwide, prospective, inception cohort study. Arthritis Res Ther 16:R101. https://doi.org/10. $1186 /$ ar4550

23. Berti A, Cornec D, Crowson CS, Specks U, Matteson EL (2017) The epidemiology of antineutrophil cytoplasmic autoantibodyassociated vasculitis in Olmsted County, Minnesota: a twentyyear US population-based study. Arthritis Rheumatol Hoboken NJ 69:2338-2350. https://doi.org/10.1002/art.40313

24. Iudici M, Pagnoux C, Quartier P, Büchler M, Cevallos R, Cohen P, de Moreuil C, Guilpain P, le Quellec A, Serratrice J, Terrier B, Guillevin L, Mouthon L, Puéchal X (2017) Childhood- versus adult-onset ANCA-associated vasculitides: a nested, matched case-control study from the French Vasculitis Study Group
Registry. Autoimmun Rev 17:108-114. https://doi.org/10.1016/j. autrev.2017.11.014

25. van der Geest KSM, Brouwer E, Sanders J-S, Sandovici M, Bos NA, Boots AMH, Abdulahad WH, Stegeman CA, Kallenberg CGM, Heeringa P, Rutgers A (2017) Towards precision medicine in ANCA-associated vasculitis. Rheumatol Oxf Engl 57:13321339. https://doi.org/10.1093/rheumatology/kex367

26. Cottin V, Bel E, Bottero P et al (2017) Revisiting the systemic vasculitis in eosinophilic granulomatosis with polyangiitis (Churg-Strauss): a study of 157 patients by the Groupe d'Etudes et de Recherche sur les Maladies Orphelines Pulmonaires and the European Respiratory Society Taskforce on eosinophilic granulomatosis with polyangiitis (Churg-Strauss). Autoimmun Rev 16:19. https://doi.org/10.1016/j.autrev.2016.09.018

27. Lanham JG, Elkon KB, Pusey CD, Hughes GR (1984) Systemic vasculitis with asthma and eosinophilia: a clinical approach to the Churg-Strauss syndrome. Medicine (Baltimore) 63:65-81

28. Jardel S, Puéchal X, Le Quellec A et al (2018) Mortality in systemic necrotizing vasculitides: a retrospective analysis of the French Vasculitis Study Group registry. Autoimmun Rev 17:653-659. https://doi.org/10.1016/j.autrev.2018.01.022

29. Tan JA, Choi HK, Xie H et al (2018) All-cause and cause-specific mortality in granulomatosis with polyangiitis: a population-based study. Arthritis Care Res. https://doi.org/10.1002/acr.23587

30. Flossmann O, Berden A, de Groot K, Hagen C, Harper L, Heijl C, Hoglund P, Jayne D, Luqmani R, Mahr A, Mukhtyar C, Pusey C, Rasmussen N, Stegeman C, Walsh M, Westman K, for the European Vasculitis Study Group (2011) Long-term patient survival in ANCA-associated vasculitis. Ann Rheum Dis 70:488-494 https://doi.org/10.1136/ard.2010.137778

31. Rowaiye OO, Kusztal M, Klinger M (2015) The kidneys and ANCA-associated vasculitis: from pathogenesis to diagnosis. Clin Kidney J 8:343-350. https://doi.org/10.1093/ckj/sfv020

32. Kubaisi B, Abu Samra K, Foster CS (2016) Granulomatosis with polyangiitis (Wegener's disease): an updated review of ocular disease manifestations. Intractable Rare Dis Res 5:61-69. https://doi. org/10.5582/irdr.2016.01014

33. Heijl C, Mohammad AJ, Westman K, Höglund P (2017) Long-term patient survival in a Swedish population-based cohort of patients with ANCA-associated vasculitis. RMD Open 3:e00435. https:// doi.org/10.1136/rmdopen-2017-000435

34. Hilhorst M, van Paassen P, Tervaert JWC, Registry LR (2015) Proteinase 3-ANCA vasculitis versus myeloperoxidase-ANCA vasculitis. J Am Soc Nephrol JASN 26:2314-2327. https://doi.org/10. 1681/ASN.2014090903

35. Hilhorst M, Wilde B, van Breda VP et al (2013) Estimating renal survival using the ANCA-associated GN classification. J Am Soc Nephrol JASN 24:1371-1375. https://doi.org/10.1681/ASN. 2012090912

36. Rathi M, Pinto B, Dhooria A, Sagar V, Mittal T, Rajan R, Dhir V, Kumar S, Sharma K, Nada R, Singh S, Minz RW, Sharma A (2016) Impact of renal involvement on survival in ANCA-associated vasculitis. Int Urol Nephrol 48:1477-1482. https://doi.org/10.1007/ s11255-016-1330-Z

Publisher's note Springer Nature remains neutral with regard to jurisdictional claims in published maps and institutional affiliations. 